\title{
Neural Representations of Self-Generated Thought during Think-aloud fMRI
}

Hui-Xian $\mathrm{Li}^{1,2,3}$, Bin $\mathrm{Lu}^{1,2,3}$, Yu-Wei Wang ${ }^{1,2,3}$, Xue-Ying $\mathrm{Li}^{1,3,5,6}$, Zhi-Chen Zhu $^{1,2,3}$, Xiao Chen ${ }^{1,2,3}$, Chao-Gan Yan ${ }^{1,2,3,4^{*}}$

${ }^{1}$ CAS Key Laboratory of Behavioral Science, Institute of Psychology, Beijing, China; ${ }^{2}$ Department of Psychology, University of Chinese Academy of Sciences, Beijing, China; ${ }^{3}$ International Big-Data Center for Depression Research, Institute of Psychology, Chinese Academy of Sciences, Beijing, China; ${ }^{4}$ Magnetic Resonance Imaging Research Center, Institute of Psychology, Chinese Academy of Sciences, Beijing, China; 5Sino-Danish College, University of Chinese Academy of Sciences, Beijing, China; ${ }^{6}$ Sino-Danish Center for Education and Research, Beijing, China.

\section{${ }^{*}$ Corresponding author:}

Chao-Gan Yan, PhD

CAS Key Laboratory of Behavioral Science, Institute of Psychology, Beijing, China

16 Lincui Road, Chaoyang District, Beijing 100101, China

Tel: +86-10-64101582 Fax: +86-10-64101582

E-mail address: yancg@psych.ac.cn (C.-G. Yan). 


\section{Abstract}

Is the brain at rest during the so-called resting-state? Ongoing experiences in the resting-state vary in unobserved and uncontrolled ways across time, individuals, and populations. However, the role of self-generated thoughts in resting-state $\mathrm{fMRI}$ remains largely unexplored. In this study, we collected realtime self-generated thoughts during resting-state fMRI scans via the think-aloud method (i.e., think-aloud fMRI), which required participants to report whatever they were currently thinking. We first investigated brain activation patterns during a think-aloud condition and found that significantly activated brain areas included all brain regions required for speech, along with a large number of deactivated brain regions. We then calculated the relationship between divergence in thought content and brain activation during think-aloud, and found that divergence in thought content was associated with a large number of brain regions, including those that were significantly activated and deactivated during think-aloud. Finally, we explored the neural representation of self-generated thoughts by performing representational similarity analysis (RSA) at three neural scales: a voxel-wise whole-brain searchlight level; a region-level whole-brain analysis using the Schaefer 400-parcels; a system level using the Yeo seven-network systems and the language subsystems. We found that resting-state self-generated thoughts were distributed across a wide range of brain regions, involving all Yeo seven-network. This study highlights the importance of taking into account ongoing experiences during resting-state fMRI, as well as providing preliminary methodological support for think-aloud fMRI.

\section{Keywords}

self-generated thoughts; think-aloud fMRI; natural language processing; representational similarity analysis 


\section{Introduction}

Resting-state functional magnetic resonance imaging (fMRI) is widely used to study normal cognitive function and to seek biological markers of clinical disease because of its relative simplicity, the ease of accumulating large data, and the ability to reveal intrinsic neural circuitry (Fox and Raichle, 2007; Mulders et al., 2015; van den Heuvel and Hulshoff Pol, 2010). Studies using resting-state $\mathrm{fMRI}$ have yielded many finding, however, these can be inconsistent and even contradictory, raising questions about the reliability of the resting-state fMRI method (Buckner et al., 2013; Cole et al., 2010; Kelly et al., 2012). Intra-individual variability in resting-state fMRI is substantial, which reduces its reliability (Zuo and Xing, 2014). The variability of unconstrained thoughts during resting-state likely plays a key role in the variability of outcome measures.

Does "resting state" fMRI data come from a "resting" brain (Finn, 2021)? Can the brain be completely at rest? Recent studies suggest that despite the absence of externally driven cognitive processing during resting-state scanning, individuals still consciously generate much ongoing endogenous cognitive processing, termed self-generated thought (Gorgolewski et al., 2014; Smallwood and Schooler, 2015). Thus, resting-state fMRI data are influenced by many factors, not only non-neural factors such as head motion and scanning conditions (Yan et al., 2009; Yan et al., 2013) but also by the ongoing experience generated by the individual during the resting-state scan and its corresponding neural activity (Buckner et al., 2013; Delamillieure et al., 2010; Gonzalez-Castillo et al., 2019). Although perception and cognition constitute individual ongoing experience at "rest," researchers rarely account for these contributions, instead applying a single term - intrinsic or spontaneous - to characterize all neuronal activity observed during resting-state fMRI (GonzalezCastillo et al., 2021; Kucyi et al., 2018; Morcom and Fletcher, 2007). However, classifying brain activity triggered by ongoing experience as simply intrinsic to the human brain blurs the signals potentially detectable from resting-state fMRI 
studies. Buckner et al. noted that while resting-state functional connectivity (FC) is not entirely constrained by anatomic connectivity, it is influenced by the mental state of the participant during scanning (Buckner et al., 2013). By comparing dynamic FC analyses between resting and anesthetized states, Barttfeld et al. found that patterns of FC during resting-state also reflected ongoing cognitive processing (Barttfeld et al., 2015); Gonzalez-Castillo et al. found that resting-state dynamic FC was influenced by short periods of spontaneous cognitive-task-like processes (Gonzalez-Castillo et al., 2019). Thus, ongoing experience (self-generated thought) during resting-state fMRI has an effect on the detected signals.

Self-generated thought is a pervasive, complex, and heterogeneous cognitive activity closely associated with various types of mental and psychiatric disorders (Andrews-Hanna et al., 2013; Christoff et al., 2016; Marchetti et al., 2016; Perkins et al., 2015; Wang et al., 2018b). Due to the variable nature of self-generated thoughts (Christoff et al., 2016), the same individual cannot be assumed to produce the same thoughts during two resting-state scans. Thus, failing to control for the effect of resting-state self-generated thoughts can significantly reduce intra-individual consistency across multiple resting-state measures, thus decreasing resting-state fMRI reliability. Another more important issue is that, for intergroup comparison studies, if groups differ in the tendency to produce self-generated thoughts, systematic intergroup differences in their resting state self-generated thoughts will likely be reflected in their resting-state neural activity patterns (Buckner et al., 2013). Notably, psychiatric patients tend to think spontaneously differently from healthy individuals (Andrews-Hanna et al., 2013; Christoff et al., 2016). For example, people with depression have a higher frequency of self-generated thoughts that have more negative content and are more related to the past compared to healthy individuals (Hoffmann et al., 2016). Self-generated thoughts in anxiety disorders are often accompanied by concerns about possible future events (Spinhoven et al., 2015); overactive and assertive self-generated thoughts are 
associated with an individual's tendency to mania (Gruber et al., 2008). Moreover, psychotic disorders are characterized by a profound disruption in self-generated thought: frequent abrupt leaps from one topic to another, or rigidity of thought, i.e., repetition or paucity of thought content (Christoff et al., 2016). If the effect of self-generated thought is not considered, the results of intergroup comparisons of resting-state fMRI between people with mental illness and healthy individuals are likely to primarily reflect differences in their self-generated thought, rather than their neural bases. Therefore, for such studies, especially the common comparison studies between patients with mental illness and healthy individuals, systematic differences in resting-state self-generated thought can affect the validity of resting-state fMRI. Therefore, the key to improving the reliability and validity of resting-state fMRI methods is to find ways to accurately measure self-generated thought and control for its effects.

It is increasingly important to reveal the ongoing experience of individuals during resting-state $\mathrm{fMRI}$ and to elucidate their effects on patterns of brain activity during resting-state scans. Answering this question would facilitate the interpretation of resting-state $\mathrm{fMRI}$ outcomes and advance the progress of resting-state MRI. However, the role of self-generated thought in resting-state fMRI remains largely unexplored and little progress has been made (GonzalezCastillo et al., 2021). Notably, research in the field of mind wandering, which has focused on task-independent cognitive processes, can provide a path for investigating self-generated thoughts in the resting state. Several brain imaging studies have linked the neural basis of mind wandering to the default mode network (DMN). Christoff et al. used the probe-capture experiment to detect when mind wandering occurred, comparing the differences in brain activity in participants when they wandered during task completion and when they did not; they found that the DMN was significantly activated but so was the executive control network during mind wandering (Christoff et al., 2009). Andrews-Hanna et al. manipulated participants' attention and found that the frequency of 
spontaneous thoughts was strongly correlated with the strength of activation of the DMN (Andrews-Hanna et al., 2010). Kucyi and Davis used the probecapture method and dynamic functional connectivity (DFC) analysis and found that DMN DFC was positively correlated with the frequency of mind wandering during task completion (Kucyi and Davis, 2014). These different approaches have implicated the DMN in spontaneous cognitive processing. Ameta-analysis of $10 \mathrm{fMRI}$ studies examining spontaneous thought processing found significant activation in several brain regions besides the DMN, including secondary somatosensory cortex, temporopolar, dorsal anterior cingulate cortex, rostrolateral prefrontal cortex, insula, and lingual gyrus, emphasizing that the neural basis of self-generated thoughts extends beyond the DMN (Fox et al., 2015). Still, although some progress has been made in identifying the correlates of self-generated thoughts, studies have mainly focused on task contexts, such as sustained attention response tasks or working memory tasks, and have rarely involved examining a task-free or resting state (Fox et al., 2015; Gonzalez-Castillo et al., 2021).

Current studies of resting-state ongoing experiences primarily use the retrospective approach in which participants are asked to assess their thoughts generated throughout the resting-state along various dimensions after completing the resting-state scan (Delamillieure et al., 2010; Diaz et al., 2014; Diaz et al., 2013; Gonzalez-Castillo et al., 2019; Gorgolewski et al., 2014; Karapanagiotidis et al., 2020; Vatansever et al., 2020; Wang et al., 2018a). Delamillieure designed the Resting State Questionnaire, which classifies experiences during resting-state scans into five categories. The investigators administered it to 180 individuals and found that the majority of participants showed a tendency to have some kind of content as the dominant mental activity, with the largest proportion of individuals reporting predominantly visual mental imagery, followed by inner language (Delamillieure et al., 2010). Gorgolewski et al. used retrospective questionnaires to measure individuals' self-generated thoughts during resting-state scanning and examined the 
association of the content and the form of thoughts with resting-state metrics. Results showed that individuals who reported more imagery exhibited greater fractional amplitude of low frequency fluctuations (fALFF) in the perigenual cingulate cortex, and individuals who reported more future thoughts exhibited lower regional homogeneity $(\mathrm{ReHo})$ in the lateral occipital cortex (Gorgolewski et al., 2014). Similarly, Wang et al. tested the correlation of certain brain states (functional connectivity, FC) with retrospective self-reports of experience during the resting-state scan and found variations along four dimensions of thought content. For example, visual experiences were associated with stronger FC between visual and other networks (Wang et al., 2018a). Although the retrospective method can inform on the relationship between general characteristics of resting-state ongoing experience and neural activity, this method lacks temporal precision and lacks individual instances that reveal ongoing mental activity, i.e., it cannot not examine real-time experience. Additionally, the retrospective method aggregates information over a long period, blurring details of content, shape, and quality of a particular event experienced in a stationary progression occurring at any given moment (i.e., the temporal blurring effect) (Gonzalez-Castillo et al., 2021). Finally, the retrospective method relies heavily on memory and has a strong lag and memory bias.

Therefore, the present study sought to collect real-time, immediate restingstate self-generated thoughts, with the goal of examining how an individual's resting-state brain activity patterns were influenced by his or her self-generated thoughts, and to clarify the direct form of brain representations of resting-state self-generated thoughts. Specifically, we used an immediate think-aloud method to collect self-generated thoughts during a resting-state fMRI scan, i.e., the think-aloud fMRI method. This method required participants to report any thoughts or images that occurred in their minds during a 10-minute "restingstate" scan. We used MRI to synchronize fMRI data throughout the process and recorded participants' verbal reports with an MRI compatible adaptive noise 
canceling microphone. Our previous research has demonstrated that the thinkaloud approach has good reliability and validity (Li et al., 2021). The verbal report did not change the characteristics of resting-state self-generated thoughts, including frequency and content characteristics. Furthermore, the metrics calculated based on natural language processing (NLP) for verbal report data had good reliability and validity. Recently, Raffaelli et al. also used the think-aloud method to quantify the content of resting-state self-generated thoughts, suggesting the ecological validity of the think-aloud approach in the application of resting-state cognition (Raffaelli et al., 2021).

A major consideration in applying the think-aloud method to MRI is whether speaking will impact brain activity. To answer this question, we first explored brain activation patterns when participants reported their thoughts. Furthermore, we designed a control condition, i.e., a cue report. The control verbal report stage was a block design, in which when participants saw the "report" cue word, they needed to report any thoughts or images that occurred in their minds during the next 20 seconds, and when they saw the "don't report" cue word, they did not need to report their thoughts during the next 20 seconds (Fig. S1). The order of reporting and non-reporting blocks was randomized. We assumed that individuals would produce a series of thoughts and mental images during both the non-reporting and reporting blocks with the only difference between the blocks being whether they spoke or not. By comparing the free reporting and non-reporting blocks we sought to examine the impact of speaking. To further illustrate a relationship between the activation of verbally reported selfgenerated thoughts and thought content, we calculated correlations between brain activation patterns and divergence in thought content using an indicator that was shown to have good reliability and validity (Li et al., 2021).

Then, we tried to detect direct neural representations of self-generated thoughts in the resting-state scan through representational similarity analysis (RSA), which can effectively clarify relationships between different data modalities (Kriegeskorte and Kievit, 2013; Popal et al., 2019). Specifically, for 
participants' verbally reported thoughts, we transcribed and labeled each selfgenerated thought episode based on topic similarity and then used NLP to convert each self-generated thought episode into a fixed-length vector for quantitative analysis. This approach has been shown to be effective for studying self-generated thoughts in the resting state (Li et al., 2021). For the fMRI data, we used the GLM to obtain the brain activation pattern of each selfgenerated thought episode. Based on the meta-analysis of spontaneous cognitive processing (Fox et al., 2015) and the diverse characteristics of resting-state experiences (Delamillieure et al., 2010), we hypothesized that self-generated thoughts during resting-state fMRI would involve a wide range of brain areas, and not only be limited to the DMN and the cognitive control network. Therefore, we examined the brain representation of resting-state selfgenerated thought using RSA at three whole-brain neural levels: a voxel-level, using whole-brain searchlight analysis; a region-level, using the Schaefer 400 parcels (Schaefer et al., 2018); and a system-level, using the seven Yeo networks (Yeo et al., 2011)(Fig. 1) and language-related networks (Xu et al., 2016).

\section{Materials and methods}

\subsection{Participants}

One hundred and one healthy adult participant recruited by internet advertisements completed the think-aloud fMRI experiment. People who reported any MRI contraindications, psychiatric or neurological disorders, use of psychotropic medications, or any history of substance or alcohol abuse were excluded. Two participants were excluded because the device was not recording. Two subjects who did not report any thoughts were excluded. Three participants whose voices were too low to be accurately transcribed were excluded. Five participants whose verbal reports contained fewer than five thoughts were excluded. Three participants were excluded because their head motion exceeded the criterion (mean Framewise Displacement (FD) Jenkinson > 
$0.2 \mathrm{~mm}$ ) (Jenkinson et al., 2002). This left a final sample size of 86 (45 females; mean age $=22.1 \pm 2.7$ years). Forty-seven completed the free verbal report and an additional control verbal report scan (24 females; mean age $=22.9 \pm$ 2.9 years). All participants gave informed written consent. All experiments were approved by the Institutional Review Board of the Institute of Psychology, Chinese Academy of Sciences.

\subsection{Experimental design}

Several days before scanning (no more than one week apart), participants were interviewed and informed of the purpose of the study and practiced producing think-aloud verbal reports outside the scanner. We briefly introduced the concept of self-generated thoughts and asked a few questions to deepen their understanding of this phenomenon. All introduction and interview information was as previously reported ( $\mathrm{Li}$ et al., 2021). We informed participants that the reporting periods would be audio-recorded throughout although we would not listen to their reports concurrently; that the audiorecording file names would not contain any personally identifiable information; and that we would only use the text for scientific research and would not disclose any of their personal information. Subsequently, participants performed a 5-minute verbal report exercise without audio recording to adapt to the think-aloud paradigm. Afterward, participants completed a 10-minute formal verbal report exercise with audio recording. Participants who participated in the control condition also performed a control verbal report condition.

All participants finished a free verbal report stage of the think-aloud fMRI method. Participants were asked to look at a fixation cross on the screen with their eyes open and stay awake and to report whatever was currently on their minds, without regard to grammar. A subset of participants completed an additional 10-minute control condition scan called the control verbal report stage (Fig. S1). This stage was a block design with two conditions. Participants 
were asked to look at the fixation cross on the screen, and when the "report" cue word appeared, they were instructed to report any thoughts or images that came to mind during the following time. When the cue word "don't report" appeared, participants did not need to make any verbal report during the subsequent time. The sequence of reporting and non-reporting phases was presented in a pseudo-randomized form. Participants completed 30 blocks in each of the two conditions. The order of the free verbal report stage and control verbal report stage was counterbalanced across participants. In addition, at the end of each stage, participants rated their thought content (temporal, social, and emotional valence) and form (words and images) on a scale from 1 to 9 points (Gorgolewski et al., 2014) (Table S1). We used FOMRI-III ${ }^{\text {TM }}$ (Fiber Optic Microphone for Functional MRI, https://www.optoacoustics.com/medical/fomriiii/features) which is an advanced adaptive noise canceling microphone used in MRI environments to record participants' verbal reports.

\subsection{MRI data acquisition}

MRI data were acquired on a GE MR750 3.0T scanner with 8-channel head coil at MRI Research Center, Institute of Psychology, Chinese Academy of Sciences. The 3D T1-weighted images were acquired using 3D-SPGR pulse sequence (192 sagittal slices, repetition time $(T R)=6.65 \mathrm{~ms}$, echo time $(T E)=$ $2.93 \mathrm{~ms}$, flip angle $(F A)=12$ degrees, inversion time $($ IT $)=450 \mathrm{~ms}$, field of view $(\mathrm{FOV})=256 \times 256 \mathrm{~mm}^{2}$, matrix size $=256 \times 256$, slice thickness $=1 \mathrm{~mm}$, voxel size $=1 \times 1 \times 1 \mathrm{~mm}^{3}$ ). The functional data were acquired with an echo-planar imaging (EPI) sequence (37 axial slices, $\mathrm{TR}=2000 \mathrm{~ms}, \mathrm{TE}=30 \mathrm{~ms}, \mathrm{FA}=90$ degrees, FOV $=224 \times 224 \mathrm{~mm}^{2}$, matrix size $=64 \times 64$, slice thickness $=3.5$ $\mathrm{mm}$, voxel size $=3.5 \times 3.5 \times 3.5 \mathrm{~mm}^{3}$ ).

As speaking-induced head motion might be a concern for the think-aloud fMRI, we adopted some strategies to control the participants' head motion. 1) Previous study reported applying a medical tape from one side of the head coil to the other side over the participant's forehead might significantly reduce head 
motion (Krause et al., 2019), we applied this strategy for almost half of our participants $(N=50)$. Although we didn't detect significant head motion difference between the participants who applied and those who didn't apply in the current study ( $p=0.591)$. 2) Padding sponges were used to fill the gap between the left and right sides of the MRI head coil and the participant' head. 3) We requested that participants move their mouths as steady as possible while speaking and emphasized the importance of controlling head motion. 4) We monitored the participants' head motion in a real time way during scanning. When participants exceeded the real time maximum head motion criteria ( $3 \mathrm{~mm}$ and 3 degrees on any axis) during the scan, we aborted the scan to restart a new scan with additional emphasizing on the need to control head motion. With all the strategies applied, we still have 3 participants whose head motion indicator mean FD_Jenkinson greater than $0.2 \mathrm{~mm}$ after postprocessing. As they exceeded the final head motion criterion, we excluded these participants in this study.

\subsection{Verbal report data preprocessing}

As in our previous study (Li et al., 2021), we converted verbal reports into text with the speech-to-text platform iFLYTEK (https://www.iflyrec.com) with manual supervision. Various stop words such as "um" and "ah" were discarded using iFLYTEK. Based on the text, we manually labeled thought switches according to the similarity of the topics and calculated the number of selfgenerated thought episodes. For example, "In the evening, I met someone to go for a walk" followed by "In a minute I'm going to call my sister" was marked as a thought switch between two different self-generated thought episodes. We confirmed the start and end time of each reported self-generated thought episode in preparation for brain imaging data analyses. Then, we mapped the sentences contained in each self-generated thought episode into a 768dimensional fixed-length vector representations using the BERT model (Devlin et al., 2019) and bert-as-service (https://github.com/hanxiao/bert-as-service). 
The pre-trained BERT model was Chinese_L-12_H-768_A-12 provided by Google.

\section{5. $\mathrm{fMRI}$ data preprocessing}

We used the toolbox DPABI (Data Processing \& Analysis for Brain Imaging, http://rfmri.org/dpabi) (Yan et al., 2016) which is based on SPM (Statistical Parametric Mapping, http://www.fil.ion.ucl.ac.uk/spm) to preprocess the fMRI data. The preprocessing was as follows: The initial 10 volumes (20 s) were removed. The slice-timing correction was performed with all volume slices corrected for different signal acquisition times by shifting the signal measured in each slice relative to the acquisition of the slice at the mid-point of each repetition time. Then, realignment was performed using a six-parameter (rigid body) linear transformation with a two-pass procedure (registered to the first image and then registered to the mean of the images after the first realignment). After realignment, individual T1-weighted MPRAGE images were co-registered to the mean functional image using a 6 degree-of-freedom linear transformation without re-sampling and then segmented into gray matter, white matter (WM), and cerebrospinal fluid (CSF). Transformations from individual native space to MNI space were computed with the Diffeomorphic Anatomical Registration Through Exponentiated Lie algebra (DARTEL) tool (Ashburner, 2007). Finally, all functional images were smoothed with a $4 \mathrm{~mm}$ FWHM Gaussian kernel.

\subsection{Analysis of activation results during the verbal report stages}

SPM12 was used for individual-level analyses; a general linear model (GLM) was estimated for each voxel. The design matrix of the free verbal report stage consists of each report and the six head motion parameters computed at the realignment stage. Here, all reported self-generated thought episodes were designed as a single condition. The design matrix of the control verbal report stage was comprised of reporting blocks, non-reporting blocks and the six head motion parameters. The regressors were convolved with the SPM canonical 
hemodynamic response function. The high-pass filter was set at $128 \mathrm{~s}$. After GLM estimation, we obtained the beta-map of reporting in the free verbal report stage. In the control verbal report stage, the contrast images of the reported and non-reported blocks were calculated. Group-level analyses were assessed using whole-brain con-weight images. Multiple comparisons were corrected using the permutation test with Threshold-Free Cluster Enhancement (TFCE) (Winkler et al., 2016). This method provides the best balance between familywise error rate and test-retest reliability (Chen et al., 2018).

\subsection{Divergence in thought content}

To test whether there was a relationship between brain activities related to self-generated thought reporting and thought content, we calculated correlations between the indicator of thought content divergence and brain activation maps. Similar to our previous study (Li et al., 2021), we first divided each 10-minute stage into two five-minute segments. Then, we calculated the sum vector of all self-generated thought episode vectors (768-dimensional token vectors from the final layer of BERT) to represent the content of the segment. Finally, we computed the inverse cosine value between two sum vectors to detect the overall divergence of thought content between the two segments (Fig. 6). This indicator represents the similarity of the text content between two segments; the smaller the value, the more similar the thought content of the two segments is.

We conducted a correlation analysis between the divergence indicator of reported thought content and the beta-map of the free verbal report stage, for which all reported thought events were organized as a single condition in the design matrix. In addition, we performed a parcel-level analysis using Schaefer 400-parcels. We averaged the activation values of all voxels within a parcel. The 400 p-values were submitted to FDR correction $(q=0.05)$.

\subsection{Representational similarity analysis on three neural scales}


We obtained the content of participants' self-generated thought episodes to construct their thought content representational dissimilarity matrix (RDM) using NLP for quantitative analysis. We also obtained each individual's BOLD fMRI responses for each self-generated thought episode. Then, neural RDMs were constructed for each individual using the thought episode pairwise correlations of BOLD response patterns at three different scales: a voxel-wise analysis with a whole-brain searchlight method; a region-level analysis with the Schaefer 400-parcels; a system level with the seven Yeo networks and language subsystems (Fig. 1).

\subsubsection{Building RDM based on the content of thought episodes}

We obtained thought RDM of reports based on BERT vectors of each selfgenerated thought episode. Specifically, the semantic distance was measured as the cosine distance between BERT vectors of each self-generated thought episode pair. Furthermore, we calculated the number of words reported for each self-generated thought episode, which reflected sentence length. The sentence length RDM was constructed by calculating the absolute difference in sentence lengths between each self-generated thought episode pair. The sentence length RDM was used to conduct RSA and used as a covariate in our main RSA analysis.

2.8.2. Obtaining whole-brain activation patterns for each reported thought episode

For each reported self-generated thought episode, a general linear model was estimated for each voxel, that is, every self-generated thought episode was considered as a separate condition. The design matrix also included the six head motion parameters. The high-pass filter was set at $128 \mathrm{~s}$. The regressors were convolved with the SPM canonical hemodynamic response function. After model estimation, the whole-brain beta-weight image for each self-generated thought episode was produced by contrasting the given report with the baseline. 


\subsubsection{Voxel-wise whole-brain searchlight analysis}

We performed a searchlight analysis (Kriegeskorte and Kievit, 2013) to determine the similarity of activation patterns across voxels between different thought episodes by using NeuroRA ( $L u$ and Ku, 2020), a python toolbox of representational analysis for multi-modal neural data. The parameters were set as follows: $k$ size $=[5,5,5]\left(\left[k_{x}, k_{y}, k_{z}\right]\right)$, Ksize refers to the number of voxels along the corresponding axes. Strides $\left.=[1,1,1]\left(\left[s_{x}, s_{y}, s_{z}\right]\right]\right)$, The Strides indicate how far the calculation unit is moved before another computation is made. Specifically, for each voxel in each participant, we extracted the activation values of neighboring voxels forming a cubic region of interest (calculation unit). Then, we constructed the neural RDM by calculating the 1 minus the spatial Pearson correlation between voxel-wise activation values of different thought episodes within this calculation unit. The neural RDM at a given voxel was compared with the thought RDM using partial Spearman correlation, in which sentence length RDM was covaried. By moving the calculation unit center throughout the cortex, we obtained whole-brain r-maps. As each voxel can exist in multiple calculation units ( $\mathrm{Lu}$ and $\mathrm{Ku}, 2020$ ), the final correlation coefficient of one voxel was the mean value of the correlation coefficients calculated by all kernels that contain that voxel (Fig. 1). For every participant, the whole-brain rmaps were Fisher's r-to-z transformed to conduct a group-level One-sample Ttest. Multiple comparisons correction was performed using the permutation test with Threshold-Free Cluster Enhancement (TFCE). Moreover, voxel-wise whole-brain searchlight analysis results were matched to the seven Yeo networks (Yeo et al., 2011) for interpretation. Specifically, we calculated the distribution of significant voxels in the Yeo networks and the ratio of significant voxels in each network.

\subsubsection{Region-level RSA}

By using the Schaefer 400-parcels (Schaefer et al., 2018), region-level 
whole-brain RSA was performed according to the following computational procedure (Fig. 1): 1) extracting the beta activation value per parcel for each thought episode; 2) computing the neural RDM as the 1 minus spatial Pearson correlation between activation values of different thought episodes for each parcel; 3) calculating the partial Spearman correlation between each parcel's RDM and thought RDM, controlling for the sentence length RDM as a covariate; and 4) transforming $r$ to $z$ using Fisher's r-to-z algorithm to conduct a grouplevel one-sample T-test. The parcel-level $p$-values were submitted to FDR correction $(q=0.05)$. Furthermore, significant parcels were categorized per the Yeo networks and the proportion of significant parcels in each network was calculated.

\subsubsection{System-level RSA}

In system-level analysis, Yeo's seven networks were used as masks for RSA (Fig. 1). The analysis process was similar to the above-mentioned regionbased approach but used network masks instead of parcels. In addition, three language modules masks (Xu et al., 2016) closely correlated with semantic processing were used to calculate RSA. The three language modules were located on the default mode network (DMN module), the left perisylvian network (PSN module), and the left frontoparietal network (FPN module). 


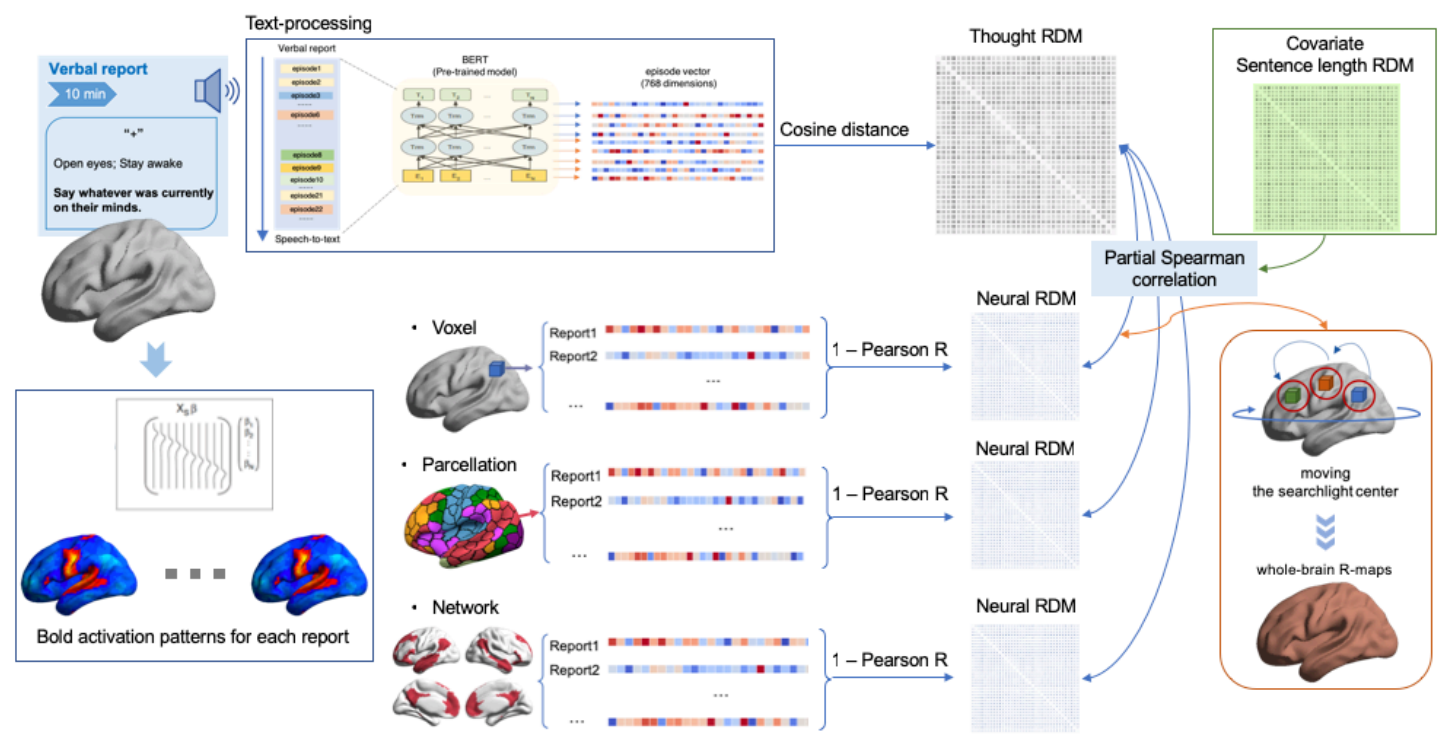

Fig. 1. The schematic diagram of RSA. The thought RDM was constructed by calculating the cosine distance of the BERT vector between two thought episodes; the sentence length RDM was constructed by calculating the absolute value of word count of two thought episodes. Brain activation maps for each reported thought episode were obtained using each thought episode as a condition. RDMs for the three neural levels were constructed by calculating the 1 minus Pearson correlation between the activation values of all voxels contained within each thought episode computational unit (voxels, parcels, and networks). The partial Spearman correlation was used to calculate the correlation between the thought RDM and the neural RDM, with the sentence length RDM as a covariate.

\subsection{Validation analysis}

We conducted supplemental analysis to confirm the validity of our results. We randomly permuted participants' thought RDMs and redid the RSA analysis to determine whether our results reflected random noise.

For head motion control, we excluded participants with mean FD_Jenkinson exceeding $0.2 \mathrm{~mm}$. Moeover, we calculated the correlation between the individual mean FD_Jenkinson index during the free verbal report stage and her/his beta-map to see if head motion influenced results.

\subsection{Data/code availability statement}


All codes are available on git-hub (https://github.com/ChaoganYan/PaperScripts/tree/master/LiHX_2022). Data have been shared online (http://rfmri.org/ SGTfMRIData). Of note, the thought vectors were shared rather than the original voice recordings to protect participants' privacy.

\section{Results}

\subsection{Report activation results}

With think-aloud $\mathrm{fMRI}$, we found that the pattern of activation of the report brain map in the free think-aloud stage (Fig. 2A) was the same as the pattern of activation of the report compared to non-report in the control verbal report stage (Fig. 2B). The control condition showed that the significantly positively activated brain areas were related to speech. Furthermore, we obtained the brain regions required for "speech" based on Neurosynth (https://www.neurosynth.org) (Fig. 2C) and found that the brain regions that were significantly positively activated in the activation pattern maps of the free speech and control stages were included in the brain regions required for "speech". It is worth noting that besides the significant activation of speechrelated brain areas, a large number of brain areas were deactivated, which may respond to thought. Therefore, we next computed the correlation between indicators of thought content divergence and brain activation. 


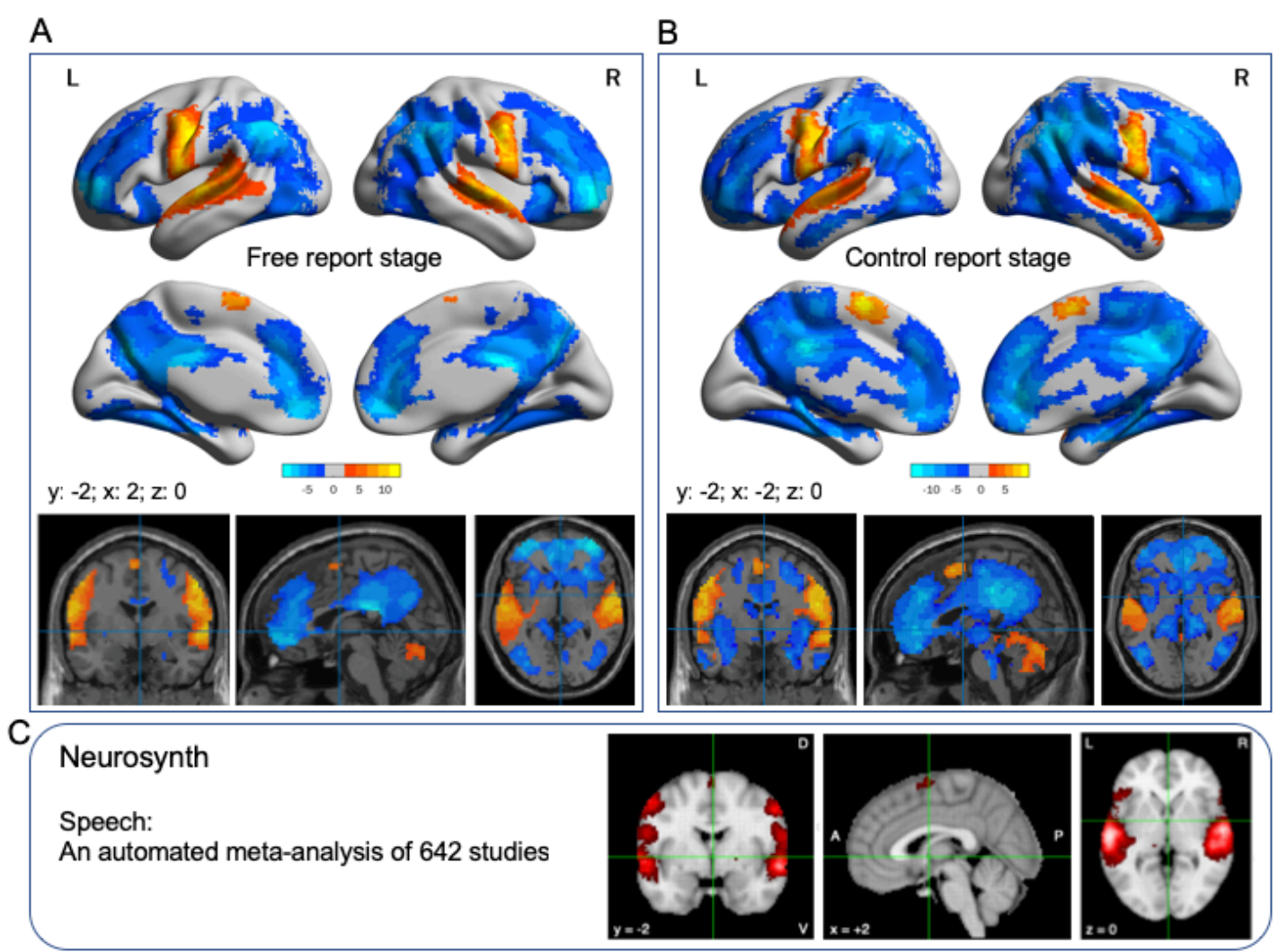

Fig. 2. Report activation results. A) The pattern of activation in the free verbal report stage.

B) The activation results of the reporting vs. non-reporting condition in the control report stage. C) Meta-analysis of 642 studies for the term "speech" based on Neurosynth.

\subsection{The results of divergence in thought content}

During the free verbal report stage, participants reported an average of 22 $(S D=10.09)$ self-generated thought episodes. The frequency distribution can be found in Fig. 3C. We used natural language processing (NLP) to calculate the divergence of thought content over time (between two five-minute segments). We then calculated the correlation between the divergence indicator and the beta-value of the free verbal report stage at the voxel and Schaefer 400-parcels levels, respectively (Fig. 3). Both results showed that the divergence of thought content was significantly correlated with a large number of brain regions (Fig. 3A, B): in addition to brain regions with significant positive activation in the reported activation results, we also detected a large number of brain regions with significant negative activation. Thus, the significantly 
deactivated brain areas in the report activation analysis reflect the content of thought.

We examined these results by reference to the corresponding Yeo networks and calculated the ratio of the number of significant voxels/parcels divided by the total number of significant voxels/parcels (Fig. 3D, F). At the voxel-level, the largest proportion of significance was found in the somatomotor network (SMN, 25.39\%), followed by the ventral attention network (VAN, $20.82 \%$ ), the DMN (19.66\%), the visual network (Visual, 15.18\%), dorsal attention network (DAN, 9.26\%), frontoparietal control network (FPCN, 8.31\%) and limbic network (Limbic, 1.38\%). The parcel results were similar to those at the voxel level.
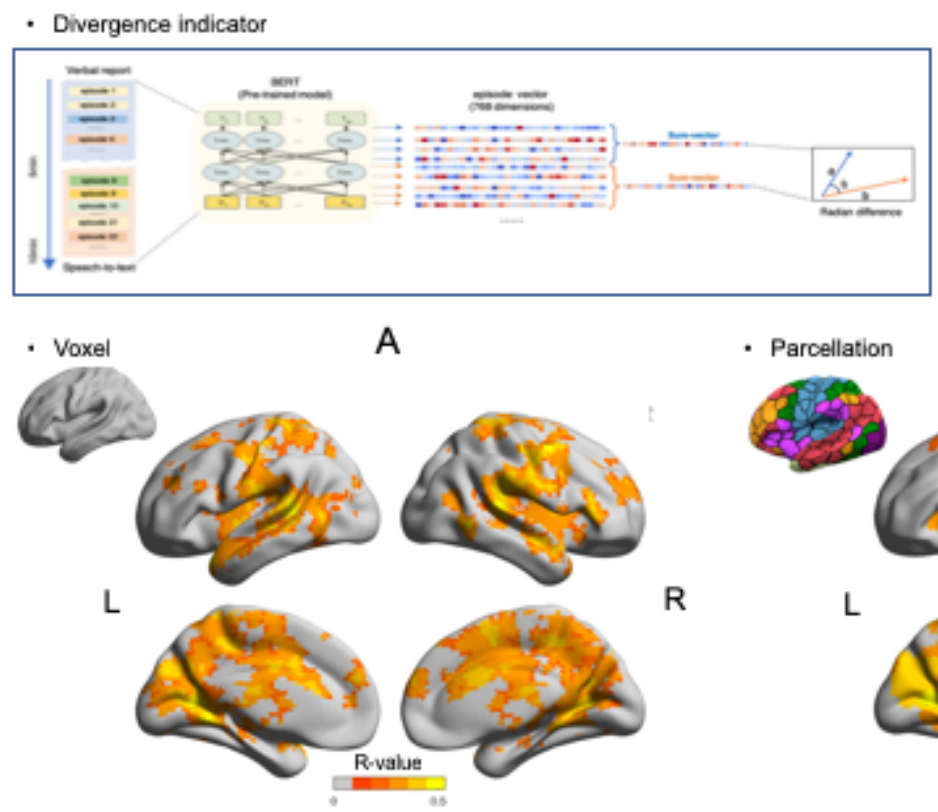

C

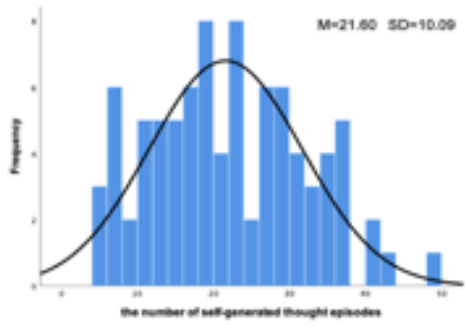

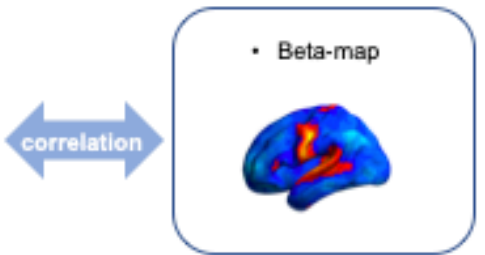

B

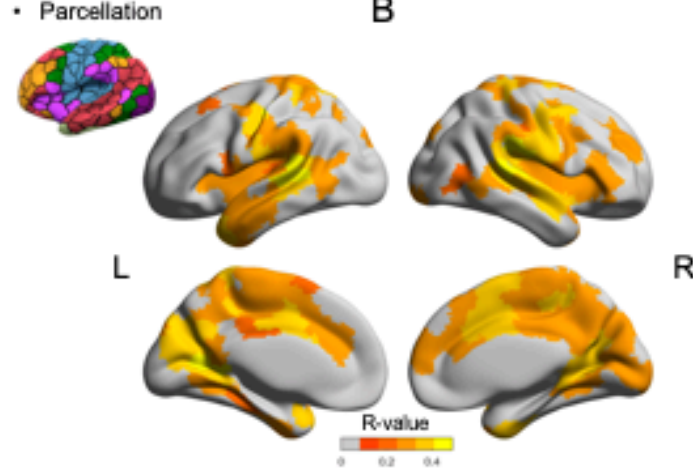

D

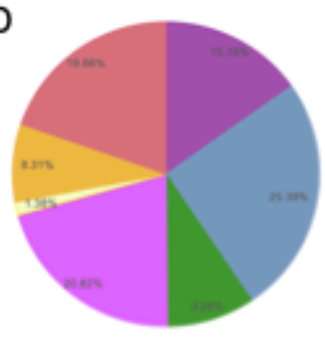

$\mathrm{F}$

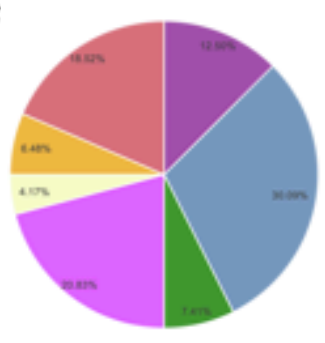

Fig. 3. Results of brain representations of divergence in thought content. Correlation results between the divergence indicator in thought content and the beta-map in the free verbal report stage based on voxels (A) and Schaefer 400-parcels (B). Multiple comparisons 
correction: Threshold-Free Cluster Enhancement (TFCE) with permutation test (Voxelwise); FDR correction, q $<0.05$ (Schaefer 400-parcels). The frequency distribution of selfgenerated thought episodes $(C)$. Significant voxels $(D)$ and parcels $(F)$ were distributed across seven Yeo networks. The ratio of the number of significant voxels/parcels divided by the total number of significant voxels/parcels.

\subsection{Representational similarity analysis results}

\subsubsection{Voxel-wise whole-brain level RSA results}

Whole-brain searchlight analysis was performed, in which neural RDMs were computed in a specific cubic centered on each voxel of the brain, and their relationships to behavioral RDMs were investigated. Given the difference in the word count of each reported self-generated thought episode, we first performed an analysis of the sentence length effect - a whole-brain searchlight using the sentence length dissimilarity matrix. The results showed a significant effect in the right insula, right superior temporal gyrus, right amygdala, right hippocampus, precuneus, and calcarine cortex (Fig. S2). Given the effect of word count, all subsequent RSAs used partial Spearman correlation, i.e., with the sentence length RDM as a covariate.

The RSA searchlight mapping for the thought RDM yielded a large number of significant regions that were widely distributed in both hemispheres (Fig. 4A) and involved all seven Yeo networks (Fig. 4B), with sentence length RDM covaried. The largest proportion of activation was in the Visual network (25.41\%), followed by the DMN (24.63\%), FPCN (15.44\%), DAN (14.08\%), VAN (11.19\%), SMN (8.39\%), and Limbic network (0.87\%) (Fig. 4C). 
A
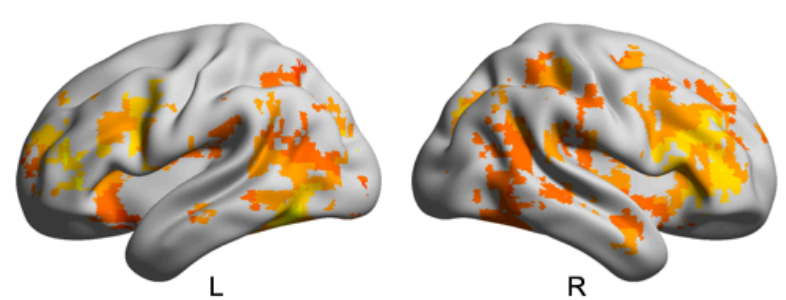

$\mathrm{R}$

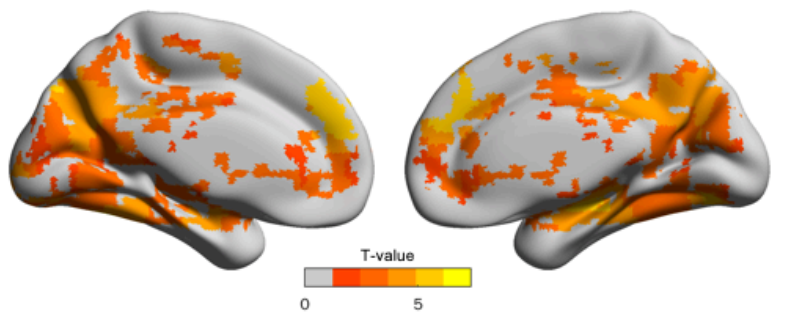

B

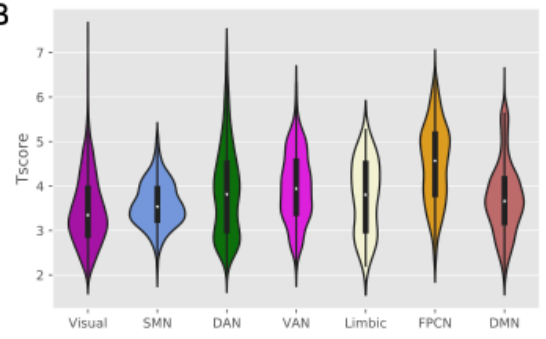

C

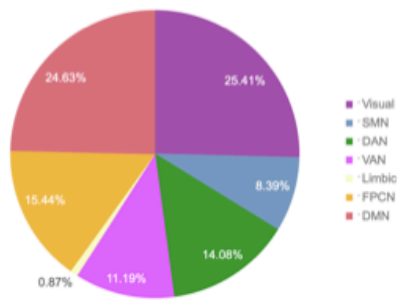

Fig. 4. RSA results of reported self-generated thought content. A) Voxel-wise searchlight showed regions whose activation patterns had a significantly positive correlation with the reported thoughts. Partial Spearman correlation was calculated by controlling for sentence length. Multiple comparison corrections: TFCE with permutation test. B) Distribution of significant voxels in the seven Yeo networks. C) Ratio of the number of significant voxels in each network. Visual: visual network; DAN: dorsal attention network; Limbic: limbic network; DMN: default mode network; SMN: somatomotor network; VAN: ventral attention network; FPCN: frontoparietal control network.

\subsubsection{Region-level RSA results}

We performed whole-brain RSA analysis based on the 400-parcels functional atlas of Schaefer et al. controlling for sentence length RDM in Spearman's partial correlation. Two hundred and eighty-three parcels survived FDR correction $(q=0.05)$, distributed throughout the Yeo networks (Fig. 5): $24.38 \%$ in the DMN, $17.67 \%$ in the SMN, $15.90 \%$ in the FPCN, $13.43 \%$ in the Visual network, $12.01 \%$ in the VAN, $9.89 \%$ in the DAN and $6.71 \%$ in the Limbic network. Even when applying strict Bonferroni correction ( $p<0.05 / 400$ ), significant parcels were still distributed over all seven Yeo networks (Fig. S3, Table S2). 
A
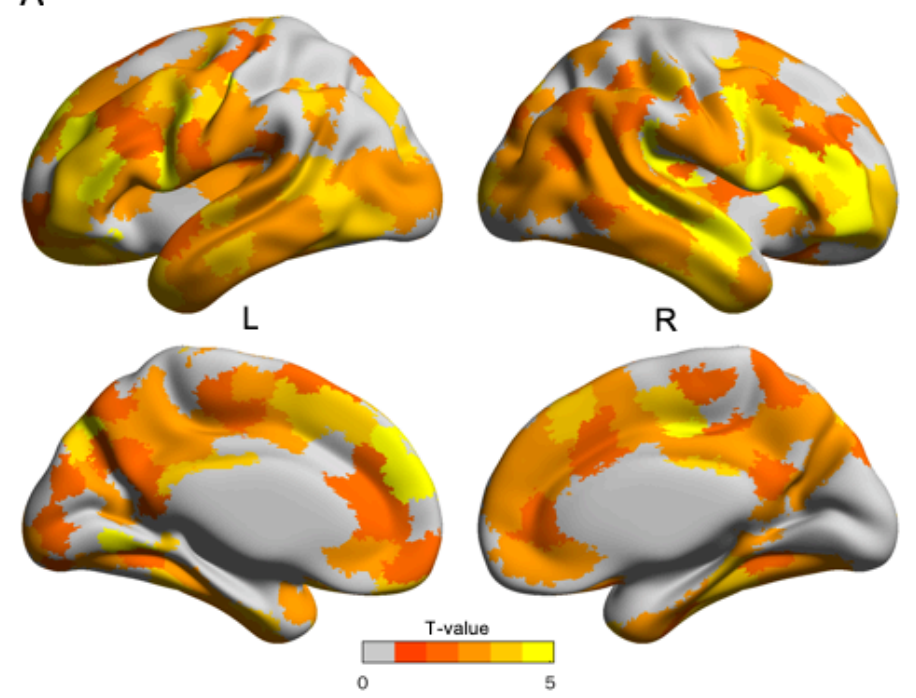

B

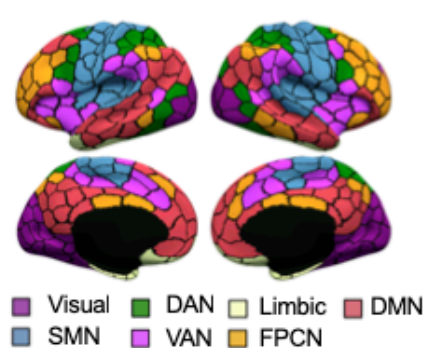

C

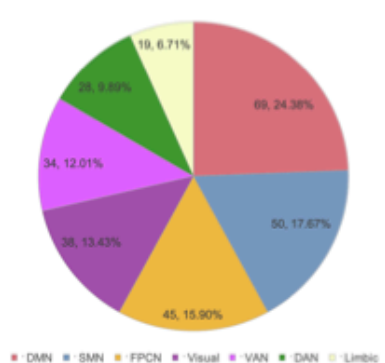

Fig. 5. RSA results based on the 400-parcel functional atlas of Schaefer et al. A) Regionwise RSA showed parcels whose activation patterns had a significantly positive correlation with the reported thought. The partial Spearmen correlation was calculated by controlling for sentence length. FDR correction was used for multiple comparisons. Results were thresholded at the $q<0.05$ level. B) 400-parcels were matched with the Yeo sevennetworks. C) The proportion of significant parcels that overlapped with the Yeo networks.

\subsubsection{System-level RSA results}

The seven Yeo network masks were used to perform RSA and all seven networks' neural RDM significantly correlated with the semantic feature RDM (Table 1). All p-values survived Bonferroni correction (0.0071).

Furthermore, we performed RSA based on language module masks which correlate closely with semantic processing, located in the default mode network, the left perisylvian network, and the left frontoparietal network. All three modules' neural RDM was significantly positively correlated with the thought RDM (Fig. S4A). The significant voxels from the whole-level searchlight RSA yielded proportions of $43.2 \%$ for the DMN Module, $38.8 \%$ for the FPN Module, and $34.6 \%$ for the PSN Module (Fig. S4C). 


\begin{tabular}{lcc}
\hline Yeo seven-networks & $\mathrm{t}$ & $\mathrm{p}$ \\
\hline Visual network & 4.688 & 0.0000104 \\
Somatomotor network & 4.877 & 0.0000050 \\
Dorsal attention network & 3.915 & 0.0001818 \\
Ventral attention network & 5.257 & 0.0000011 \\
Limbic network & 2.838 & 0.0056813 \\
Frontoparietal control network & 5.554 & 0.0000003 \\
Default mode network & 5.327 & 0.0000008 \\
\hline
\end{tabular}

Note: Sentence length RDM as a covariate. Bonferroni correction $p<0.05 / 7$.

\subsection{Validation analysis}

Supplemental validation analyses were performed using the randomly permuted thought RDM. We randomly permuted the order of participants' reported thought episodes to construct the semantic RDM with random order. Then this RDM was used to perform voxel-wise-level searchlight RSA, regionlevel RSA, and system-level RSA, respectively. We did not find any significant results in any of the levels of analysis.

Most participants' head motion $(n=101, M=0.095, S D=0.045)$ during the verbal report stage were below the criterion (mean FD_Jenkinson $<0.2 \mathrm{~mm}$ ) (Fig. S5). Three participants with greater head motion were excluded. We also conducted a correlation between head motion and the verbal report stage betavalue. No brain regions were significantly correlated with head motion.

\section{Discussion}

Investigators have long suggested that considering ongoing experiences during the resting-state is important for the development of resting-state fMRI, however, few studies investigated self-generated thoughts directly. Here, we used the think-aloud fMRI method to collect participants' real-time self- 
generated thoughts during "resting-state" scans and then used NLP to quantify each self-generated thought episode into a 768-dimension fixed-length vector to construct semantic feature RDM. To clarify how resting-state self-generated thoughts manifest in the brain, we investigated the neural correlations between reported thoughts and their corresponding brain activation by RSA at three scales, at the levels of voxels, regions and systems. Our main finding was that resting-state self-generated thoughts involved a wide range of brain regions across all seven Yeo networks.

One of the factors that may account for the modest reliability of restingstate $\mathrm{fMRI}$ is the inherent complexity of ongoing mental activity during scanning (Morcom and Fletcher, 2007). The classical resting-state fMRI design is simple ("look at the '+' fixation point and stay awake" or "close your eyes and rest"), making it difficult to influence and determine participants' mental states during scans (Power et al., 2014). Participant self-generated thoughts may include planning the future, recalling the past, reflecting, and ruminating. Clearly, the so-called "resting-state" is not a complete standstill of the brain (Buckner, 2012), but rather reflects a mixture of mental activity and intrinsic functional organization (Finn, 2021). Likely because we pay little attention to observing these ongoing mental activities during resting-state scans, the interpretability of resting-state $\mathrm{fMRI}$ is inferior (Gonzalez-Castillo et al., 2021). Resting-state results cannot be simply attributed to the brain's intrinsic activity. Thus, examining ongoing experiences during resting-state scans is increasingly important. However, such research has largely relied on retrospective introspection in which participants were asked to assess the characteristics of self-generated thoughts in certain dimensions throughout the resting-state scan. This method provides a window into ongoing experiences in the resting-state but because of methodological limitations, we are still unable to definitively study self-generated thoughts during resting-state scans. In this study, we used an immediate think-aloud fMRI approach to collect real-time ongoing experiences during "resting-state" scanning. Moreover, we combined NLP to 
directly quantitatively analyze self-generated thoughts, avoiding the lag of retrospective methods and reducing introspection, as well as expanding the study of the content features of self-generated thoughts (open to exploration without being limited to predefined thought dimensional characteristics).

However, a major consideration in applying the think-aloud method to MRI relates to the potential of speaking to increase head motion, and to the impact of speaking on brain activity. We took some measures to control participants' head motion (see materials and methods). We found that most participants were able to control themselves well, with only 3 out of 101 participants having head motion that exceeded criterion (mean FD_Jenkinson $<0.2 \mathrm{~mm}$ ) (Fig. S5). In addition, we calculated correlations between our head motion index (mean FD_Jenkinson) and individual brain activations (beta-maps) and did not find any brain regions significantly associated with individual head motion. As to whether speaking has a significant effect on individual brain activity, we expected individuals would significantly activate brain areas associated with speech when they reported their thoughts (Fig. 2). Accordingly, we designed a control condition, i.e., cue report (Fig. S1). In this block design, the nonreporting phase is the classical resting-state and the reporting phase is the immediate think-aloud phase. We hypothesized that individuals would produce a series of thoughts and images in their minds during both the non-reporting and reporting phases and that the only difference between the two phases would be whether they spoke or not. In comparing the reporting phase with the non-reporting phase, we found significant activation of language-related networks (Fig. 2). This brain pattern is largely consistent with that of the free verbal report stage and overlapped with the brain areas associated with "speech" in Neurosynth. Notably, in addition to the positively activated language-related brain areas, we found extensive deactivated brain areas, which we suspected could be associated with self-generated thoughts. Therefore, we calculated the correlations between thought content divergence and brain activation. Results were distributed across a wide range of 
deactivated brain areas, suggesting that these brain areas were involved in thought (Fig. 3).

We computed neural response patterns of self-generated thoughts in the resting-state fMRI using RSA on three neural scales and found that the representation of self-generated thoughts in the resting-state is diffuse: it is not just the DMN that characterizes self-generated thoughts in the resting-state but involves a wide range of regions throughout the brain, including all seven Yeo networks. This result confirms previous findings. A meta-analysis by Fox et al. showed that spontaneous thought processes were significantly correlated with the DMN, nevertheless, although a large number of non-DMN regions also had consistent activation such as secondary somatosensory cortices, lingual gyrus, and insula. Thus, they emphasized that the DMN was not sufficient to adequately capture the neural basis of self-generated cognitive process and that regions of non-DMN networks, such as FPCN, were as important to spontaneous thoughts as the DMN (Fox et al., 2015). Moreover, the functional connectivity (FC) between different networks makes a difference to the characteristics of self-generated thoughts at rest. Wang et al. calculated the correlation between self-generated thoughts during resting-state scans and large-scale network organization based on the retrospective self-report method (Wang et al., 2018a). The results showed that the four different dimensions of self-generated thought at rest correlated differently with different FCs. For example, experiences focused on "personal importance" were associated with reduced FC within the attention and control system, while "purposeful" experiences were correlated with lower FC between DMN and limbic network.

Self-generated thought at rest is complex and variable (Wang et al., 2018b), its form includes images, words, etc., and its content includes temporal dimensions, social dimensions, emotional valence, etc. (Gorgolewski et al., 2014). In this study, we found a substantial proportion of significant voxels in the visual network, which is consistent with individuals reporting that much of inner experience in the resting state involves visual imagery (seeing something 
in thought) (Delamillieure et al., 2010). Relatedly, retrospective assessment after the free verbal report stage scan showed that participants related many visual imagery experiences (Fig. S6, Table S3). Visual networks have an important role in visual imagery (Ganis et al., 2004), and researchers have demonstrated that visual areas are associated with the emergence of visual, non-verbal features of self-generated thoughts; moreover, visual thoughts were associated with more positive valence (Raij and Riekki, 2017). Except for visual thoughts, a large portion of self-generated thoughts is in the form of internal verbal (thinking in words without overt vocal production) (Delamillieure et al., 2010; Raij and Riekki, 2017). We used brain regions closely related to semantic processing based on resting-state (Xu et al., 2016) (Fig. S4A), and significant voxels based on voxel-level searchlight RSA fell in all three modules (Fig. S4B), with the DNM module accounting for more than half of them $(50.45 \%)$, followed by the PSN module (32.98\%) and the FPN module (16.57\%)(Fig. S4C). Meanwhile, the RSA results based on three modules showed significant correlations between the three networks and the reported thoughts (Fig. S4A).

The activation of the attention network including DAN and VAN may reflect the transition between internally driven and externally driven resting-state experience. Although there was no explicit external task during the resting-state scan, scanner noise was still present and participants still needed to keep their eyes open to gaze at the fixation point. Self-generated thoughts focused on "personal importance" were associated with the attention network (Wang et al., 2018a). The SMN may represent an individual's processing of external and internal sensations, including scanner noise and self-body somatomotor experiences. Moreover, the somatomotor network was important for language processing (Pulvermüller and Fadiga, 2010). Finally, the limbic network embodies emotional experience in self-generated thoughts at rest. Selfgenerated thoughts involve emotional information and are linked to mood state (Andrews-Hanna et al., 2013; Killingsworth and Gilbert, 2010; Ruby et al., 2013), such that healthy individuals think about more positive things while depressed 
individuals think about more negative events. Furthermore, the FC of the limbic network to other networks was found to be related to different forms of experiences at rest. For example, emotional experiences were associated with higher FC between limbic and VAN; visual experiences were associated with stronger connectivity between visual and limbic networks (Wang et al., 2018a).

\section{Limitation and future directions}

The current study directly quantifies the form of brain representation of selfgenerated thoughts during resting-state $\mathrm{fMRI}$, however, it is unclear how the different content features of self-generated thoughts relate specifically to the corresponding networks. Addressing this aspect will requires clarifying the specific characteristics of each reported thought, such as whether it belongs to past event recall or body sensation, etc. Based on reported self-generated thought episodes with labels will help us to clarify the specific brain activation patterns corresponding to different experiences types. Furthermore, it is necessary to obtain real-time self-generated thoughts data during resting-state scans across different psychiatric disorders, which will help us to clarify how to control for the impact of self-generated thoughts when comparing between groups, or to better interpret resting-state fMRI results using self-generated thoughts. The current study is a preliminary exploration, with more research needed to explore in-depth the characteristics of ongoing experiences in the resting-state fMRI.

\section{Conclusion}

In conclusion, we directly and quantitatively demonstrated that selfgenerated thoughts during think-aloud fMRI were closely associated with a wide range of brain regions. This study highlights the need to account for the characteristics of the self-generated thoughts when using resting-state fMRI. In particular, differences in resting-state fMRI self-generated thoughts should be considered when comparing groups, especially for groups with psychiatric 
disorders.

\section{Declaration of competing interest}

The authors declare no conflicts of interest.

\section{Credit authorship contribution statement}

Hui-Xian Li: Conceptualization, Methodology, Software, Formal analysis, Investigation, Data Curation, Writing - Original Draft, Writing - Review \& Editing, Visualization, Funding acquisition. Bin Lu: Methodology, Investigation. Yu-Wei Wang: Investigation. Xue-Ying Li: Investigation. Zhi-Chen Zhu: Investigation. Chao-Gan Yan: Conceptualization, Methodology, Software, Writing - Original Draft, Writing - Review \& Editing, Supervision, Project administration, Funding acquisition.

\section{Acknowledgment}

We thank Dr. Francisco Xavier Castellanos for his comments and edits on our work. We appreciate Dr. Yanchao Bi for her comments and generously sharing their semantic masks. This work was supported by the National Natural Science Foundation of China $(82122035,81671774,81630031)$, the Key Research Program of the Chinese Academy of Sciences (ZDBS-SSW-JSC006), Beijing Nova Program of Science and Technology (Z191100001119104). 


\section{References}

Andrews-Hanna, J.R., Kaiser, R.H., Turner, A.E., Reineberg, A.E., Godinez, D., Dimidjian, S., Banich, M.T., 2013. A penny for your thoughts: dimensions of selfgenerated thought content and relationships with individual differences in emotional wellbeing. Frontiers in Psychology 4, 900.

Andrews-Hanna, J.R., Reidler, J.S., Huang, C., Buckner, R.L., 2010. Evidence for the default network's role in spontaneous cognition. Journal of Neurophysiology $104,322-335$.

Ashburner, J., 2007. A fast diffeomorphic image registration algorithm. Neuroimage 38, 95-113.

Barttfeld, P., Uhrig, L., Sitt, J.D., Sigman, M., Jarraya, B., Dehaene, S., 2015. Signature of consciousness in the dynamics of resting-state brain activity. Proceedings of the National Academy of Sciences of the United States of America 112, 887-892.

Buckner, R.L., 2012. The serendipitous discovery of the brain's default network. Neuroimage 62, 1137-1145.

Buckner, R.L., Krienen, F.M., Yeo, B.T., 2013. Opportunities and limitations of intrinsic functional connectivity MRI. Nat Neurosci 16, 832-837.

Chen, X., Lu, B., Yan, C.G., 2018. Reproducibility of R-fMRI metrics on the impact of different strategies for multiple comparison correction and sample sizes. Hum Brain Mapp 39, 300-318.

Christoff, K., Gordon, A.M., Smallwood, J., Smith, R., Schooler, J.W., 2009. 
Experience sampling during fMRI reveals default network and executive system contributions to mind wandering. Proceedings of the National Academy of Sciences of the United States of America 106, 8719-8724.

Christoff, K., Irving, Z.C., Fox, K.C., Spreng, R.N., Andrews-Hanna, J.R., 2016. Mind-wandering as spontaneous thought: a dynamic framework. Nat Rev Neurosci 17, 718-731.

Cole, D.M., Smith, S.M., Beckmann, C.F., 2010. Advances and pitfalls in the analysis and interpretation of resting-state FMRI data. Front Syst Neurosci 4, 8.

Delamillieure, P., Doucet, G., Mazoyer, B., Turbelin, M.R., Delcroix, N., Mellet, E., Zago, L., Crivello, F., Petit, L., Tzourio-Mazoyer, N., Joliot, M., 2010. The resting state questionnaire: An introspective questionnaire for evaluation of inner experience during the conscious resting state. Brain Research Bulletin 81, 565-573.

Devlin, J., Chang, M.-W., Lee, K., Toutanova, K., 2019. BERT: Pre-training of Deep Bidirectional Transformers for Language Understanding. Association for Computational Linguistics, 4171-4186.

Diaz, B.A., Van Der Sluis, S., Benjamins, J.S., Stoffers, D., Hardstone, R., Mansvelder, H.D., Van Someren, E.J., Linkenkaer-Hansen, K., 2014. The ARSQ 2.0 reveals age and personality effects on mind-wandering experiences. Frontiers in Psychology 5, 271.

Diaz, B.A., Van Der Sluis, S., Moens, S., Benjamins, J.S., Migliorati, F., Stoffers, D., Den Braber, A., Poil, S.S., Hardstone, R., Van't Ent, D., Boomsma, D.I., De Geus, E., Mansvelder, H.D., Van Someren, E.J., Linkenkaer-Hansen, K., 2013. The 
Amsterdam Resting-State Questionnaire reveals multiple phenotypes of restingstate cognition. Front Hum Neurosci 7, 446.

Finn, E.S., 2021. Is it time to put rest to rest? Trends in Cognitive Sciences 25, $1021-1032$.

Fox, K.C., Spreng, R.N., Ellamil, M., Andrews-Hanna, J.R., Christoff, K., 2015. The wandering brain: meta-analysis of functional neuroimaging studies of mindwandering and related spontaneous thought processes. Neuroimage 111, $611-$ 621.

Fox, M.D., Raichle, M.E., 2007. Spontaneous fluctuations in brain activity observed with functional magnetic resonance imaging. Nature Reviews: Neuroscience 8, 700-711.

Ganis, G., Thompson, W.L., Kosslyn, S.M., 2004. Brain areas underlying visual mental imagery and visual perception: an fMRI study. Brain Research: Cognitive Brain Research 20, 226-241.

Gonzalez-Castillo, J., Caballero-Gaudes, C., Topolski, N., Handwerker, D.A., Pereira, F., Bandettini, P.A., 2019. Imaging the spontaneous flow of thought: Distinct periods of cognition contribute to dynamic functional connectivity during rest. Neuroimage 202, 116129.

Gonzalez-Castillo, J., Kam, J.W.Y., Hoy, C.W., Bandettini, P.A., 2021. How to Interpret Resting-State fMRI: Ask Your Participants. Journal of Neuroscience 41, $1130-1141$.

Gorgolewski, K.J., Lurie, D., Urchs, S., Kipping, J.A., Craddock, R.C., Milham, 
M.P., Margulies, D.S., Smallwood, J., 2014. A correspondence between individual differences in the brain's intrinsic functional architecture and the content and form of self-generated thoughts. Plos One 9, e97176.

Gruber, J., Johnson, S.L., Oveis, C., Keltner, D., 2008. Risk for mania and positive emotional responding: too much of a good thing? Emotion 8, 23-33.

Hoffmann, F., Banzhaf, C., Kanske, P., Bermpohl, F., Singer, T., 2016. Where the depressed mind wanders: Self-generated thought patterns as assessed through experience sampling as a state marker of depression. Journal of Affective Disorders 198, 127-134.

Jenkinson, M., Bannister, P., Brady, M., Smith, S., 2002. Improved optimization for the robust and accurate linear registration and motion correction of brain images. Neuroimage 17, 825-841.

Karapanagiotidis, T., Vidaurre, D., Quinn, A.J., Vatansever, D., Poerio, G.L., Turnbull, A., Ho, N.S.P., Leech, R., Bernhardt, B.C., Jefferies, E., Margulies, D.S., Nichols, T.E., Woolrich, M.W., Smallwood, J., 2020. The psychological correlates of distinct neural states occurring during wakeful rest. Scientific Reports 10, 21121.

Kelly, C., Biswal, B.B., Craddock, R.C., Castellanos, F.X., Milham, M.P., 2012. Characterizing variation in the functional connectome: promise and pitfalls. Trends Cogn Sci 16, 181-188.

Killingsworth, M.A., Gilbert, D.T., 2010. A wandering mind is an unhappy mind. Science 330, 932.

Krause, F., Benjamins, C., Eck, J., Luhrs, M., van Hoof, R., Goebel, R., 2019. 
Active head motion reduction in magnetic resonance imaging using tactile feedback. Hum Brain Mapp 40, 4026-4037.

Kriegeskorte, N., Kievit, R.A., 2013. Representational geometry: integrating cognition, computation, and the brain. Trends in Cognitive Sciences 17, 401-412.

Kucyi, A., Davis, K.D., 2014. Dynamic functional connectivity of the default mode network tracks daydreaming. Neuroimage 100, 471-480.

Kucyi, A., Tambini, A., Sadaghiani, S., Keilholz, S., Cohen, J.R., 2018. Spontaneous cognitive processes and the behavioral validation of time-varying brain connectivity. Network neuroscience 2, 397-417.

Li, H.X., Lu, B., Chen, X., Li, X.Y., Castellanos, F.X., Yan, C.G., 2021. Exploring self-generated thoughts in a resting state with natural language processing. Behav Res Methods.

Lu, Z., Ku, Y., 2020. NeuroRA: A Python Toolbox of Representational Analysis From Multi-Modal Neural Data. Frontiers in Neuroinformatics 14, 563669.

Marchetti, I., Koster, E.H.W., Klinger, E., Alloy, L.B., 2016. Spontaneous Thought and Vulnerability to Mood Disorders: The Dark Side of the Wandering Mind. Clinical psychological science 4, 835-857.

Morcom, A.M., Fletcher, P.C., 2007. Does the brain have a baseline? Why we should be resisting a rest. Neuroimage 37, 1073-1082.

Mulders, P.C., van Eijndhoven, P.F., Schene, A.H., Beckmann, C.F., Tendolkar, I., 2015. Resting-state functional connectivity in major depressive disorder: A review. Neuroscience and biobehavioral reviews 56, 330-344. 
Perkins, A.M., Arnone, D., Smallwood, J., Mobbs, D., 2015. Thinking too much: self-generated thought as the engine of neuroticism. Trends in Cognitive Sciences 19, 492-498.

Popal, H., Wang, Y., Olson, I.R., 2019. A Guide to Representational Similarity Analysis for Social Neuroscience. Social Cognitive and Affective Neuroscience 14, $1243-1253$.

Power, J.D., Schlaggar, B.L., Petersen, S.E., 2014. Studying brain organization via spontaneous fMRI signal. Neuron 84, 681-696.

Pulvermüller, F., Fadiga, L., 2010. Active perception: sensorimotor circuits as a cortical basis for language. Nature Reviews: Neuroscience 11, 351-360.

Raffaelli, Q., Mills, C., de Stefano, N.A., Mehl, M.R., Chambers, K., Fitzgerald, S.A., Wilcox, R., Christoff, K., Andrews, E.S., Grilli, M.D., O'Connor, M.F., AndrewsHanna, J.R., 2021. The think aloud paradigm reveals differences in the content, dynamics and conceptual scope of resting state thought in trait brooding. Scientific Reports 11, 19362.

Raij, T.T., Riekki, T.J.J., 2017. Dorsomedial Prefontal Cortex Supports Spontaneous Thinking Per Se. Human Brain Mapping 38, 3277-3288.

Ruby, F.J., Smallwood, J., Engen, H., Singer, T., 2013. How self-generated thought shapes mood--the relation between mind-wandering and mood depends on the socio-temporal content of thoughts. PloS One 8, e77554.

Schaefer, A., Kong, R., Gordon, E.M., Laumann, T.O., Zuo, X.N., Holmes, A.J., Eickhoff, S.B., Yeo, B.T.T., 2018. Local-Global Parcellation of the Human Cerebral 
Cortex from Intrinsic Functional Connectivity MRI. Cerebral Cortex 28, 3095-3114.

Smallwood, J., Schooler, J.W., 2015. The science of mind wandering: empirically navigating the stream of consciousness. Annu Rev Psychol 66, 487518.

Spinhoven, P., Drost, J., van Hemert, B., Penninx, B.W., 2015. Common rather than unique aspects of repetitive negative thinking are related to depressive and anxiety disorders and symptoms. Journal of Anxiety Disorders 33, 45-52.

van den Heuvel, M.P., Hulshoff Pol, H.E., 2010. Exploring the brain network: a review on resting-state fMRI functional connectivity. European Neuropsychopharmacology 20, 519-534.

Vatansever, D., Karapanagiotidis, T., Margulies, D.S., Jefferies, E., Smallwood, J., 2020. Distinct patterns of thought mediate the link between brain functional connectomes and well-being. Network neuroscience 4, 637-657.

Wang, H.T., Bzdok, D., Margulies, D., Craddock, C., Milham, M., Jefferies, E., Smallwood, J., 2018a. Patterns of thought: Population variation in the associations between large-scale network organisation and self-reported experiences at rest. Neuroimage 176, 518-527.

Wang, H.T., Poerio, G., Murphy, C., Bzdok, D., Jefferies, E., Smallwood, J., 2018b. Dimensions of Experience: Exploring the Heterogeneity of the Wandering Mind. Psychological Science 29, 56-71.

Winkler, A.M., Ridgway, G.R., Douaud, G., Nichols, T.E., Smith, S.M., 2016. Faster permutation inference in brain imaging. Neuroimage 141, 502-516. 
Xu, Y., Lin, Q., Han, Z., He, Y., Bi, Y., 2016. Intrinsic functional network architecture of human semantic processing: Modules and hubs. Neuroimage 132, $542-555$.

Yan, C., Liu, D., He, Y., Zou, Q., Zhu, C., Zuo, X., Long, X., Zang, Y., 2009. Spontaneous brain activity in the default mode network is sensitive to different resting-state conditions with limited cognitive load. PLoS ONE 4, e5743.

Yan, C.G., Cheung, B., Kelly, C., Colcombe, S., Craddock, R.C., Di Martino, A., Li, Q., Zuo, X.N., Castellanos, F.X., Milham, M.P., 2013. A comprehensive assessment of regional variation in the impact of head micromovements on functional connectomics. Neuroimage 76, 183-201.

Yan, C.G., Wang, X.D., Zuo, X.N., Zang, Y.F., 2016. DPABI: Data Processing \& Analysis for (Resting-State) Brain Imaging. Neuroinformatics 14, 339-351.

Yeo, B.T., Krienen, F.M., Sepulcre, J., Sabuncu, M.R., Lashkari, D., Hollinshead, M., Roffman, J.L., Smoller, J.W., Zollei, L., Polimeni, J.R., Fischl, B., Liu, H., Buckner, R.L., 2011. The organization of the human cerebral cortex estimated by intrinsic functional connectivity. J Neurophysiol 106, 1125-1165.

Zuo, X.N., Xing, X.X., 2014. Test-retest reliabilities of resting-state FMRI measurements in human brain functional connectomics: a systems neuroscience perspective. Neuroscience and Biobehavioral Reviews 45, 100-118. 